\title{
Análisis de pH y concentración de peróxido de hidrógeno en geles blanqueadores de uso profesional
}

\author{
Analysis of $\mathrm{pH}$ and hydrogen peroxide concentration in professional bleaching gels \\ Matías Mederos ${ }^{a}$, Carlos E. Cuevas-Suarez, ${ }^{b}$, \\ Alejandro Francia ${ }^{c}$ y Guillermo Grazioli ${ }^{d}$
}

\begin{abstract}
:
The objective of the present in vitro study was to measure the $\mathrm{pH}$ and hydrogen peroxide concentration of professional bleaching gels and to compare these values with those specified by the manufacturer. Different bleaching agents for professional use whose active agent is hydrogen peroxide in high concentrations were selected. The $\mathrm{pH}$ and hydrogen peroxide concentration were analyzed in accordance with the international standard ISO 28399 and the American Dental Association Standard for bleaching agents. In the analysis of the results of the $\mathrm{pH}$ measurements, statistically significant differences were found between the values of all the products analyzed. All the groups showed a statistically significant decrease in the $\mathrm{H}_{2} \mathrm{O}_{2}$ concentration measured experimentally, with respect to that specified by their respective manufacturer. The discrepancies observed could affect the whitening efficacy expected for both patient and dentist, and must be considered when establishing a treatment plan.
\end{abstract}

Keywords:

hydrogen peroxide, $\mathrm{pH}$, bleaching agent.

\section{Resumen:}

El objetivo del presente estudio in vitro, fue medir el pH y la concentración de peróxido de hidrogeno de geles blanqueadores de uso profesional y comparar dichos valores con los especificados por el fabricante. Fueron seleccionados diferentes agentes blanqueadores de uso profesional cuyo agente activo es el peróxido de hidrógeno en altas concentraciones. $\mathrm{El} \mathrm{pH}$ y la concentración de peróxido de hidrógeno fueron analizados de acuerdo con lo establecido en la norma internacional ISO 28399 y la norma para agentes blanqueadores de la Asociación Dental Americana. En el análisis de los resultados de las mediciones de $\mathrm{pH}$ se encontraron diferencias estadísticamente significativas entre los valores de todos los productos analizados. Todos los grupos mostraron una disminución estadísticamente significativa en la concentración de $\mathrm{H}_{2} \mathrm{O}_{2}$ medida experimentalmente, con respecto a la especificada por su respectivo fabricante. Las discrepancias observadas pueden afectar la eficacia de blanqueamiento esperada tanto por el paciente como por el dentista, y debe ser considerado a la hora de establecer un plan de tratamiento.

\section{Palabras Clave:}

peróxido de hidrogeno, $\mathrm{pH}$, agentes blanqueadores.

\footnotetext{
${ }^{a}$ Cátedra de Materiales Dentales, Facultad de Odontología, UdelaR, Uruguay. https://orcid.org/0000-0002-1561-2283,

Email: matiasmederos@odon.edu.uy

${ }^{\mathrm{b}}$ Laboratorio de Materiales Dentales. Universidad Autónoma del Estado de Hidalgo, Instituto de Ciencias de la Salud, https://orcid.org/00000002-2759-8984, Email: cecuevas@uaeh.edu.mx

c Cátedra de Fisiología general y bucodental, Facultad de Odontología, UdelaR, Uruguay. https://orcid.org/0000-0002-7942-9189, Email: ale-fp@hotmail.com

d Autor de Correspondencia, Cátedra de Materiales Dentales, Facultad de Odontología, UdelaR, Uruguay. https://orcid.org/0000-0001-99693780, Email: ggrazioli@odon.edu.uy
} 


\section{Introducción}

Dada la creciente demanda de salud y estética, cada vez más pacientes solicitan tratamiento para la decoloración dental.1,2 Entre las terapias actualmente disponibles, el blanqueamiento en dientes vitales mediante el uso de agentes blanqueadores representa un enfoque conservador.3,4 Este procedimiento se puede realizar en diferentes momentos, por el profesional en el consultorio (in-office bleaching), por el propio paciente en su domicilio con supervisión del profesional (at-home bleaching), sin supervisión profesional con productos disponibles en el mostrador (over-the-counter products), o combinados.5,6 Generalmente, los productos con altas concentraciones del componente "activo" son aplicados por el profesional, mientras que los de bajas concentraciones son aplicados por el paciente en el hogar. En ambas estrategias, el componente activo es generalmente peróxido de hidrógeno o peróxido de carbamida.6-8

Es importante destacar que el peróxido de hidrógeno es una sustancia químicamente inestable, siendo diversos los factores que pueden afectar dicha estabilidad, tales como la temperatura, 9 el tiempo de almacenamiento, 9 la presencia de contaminantes o impurezas, 10,11 y el pH del medio,12,13 y se ha sugerido que los mismos podrían influir en la concentración de peróxido de hidrógeno disponible al momento de su aplicación. Diversos estudios han demostrado que además del tiempo de aplicación, la concentración de peróxido de hidrógeno es una de las variables que afectan la eficacia del blanqueamiento,1416 y que el efecto blanqueador deseado es inducido por este agente químico y no por la acidez del producto.17,18 En este sentido, aún se presentan en el mercado productos con valores de $\mathrm{pH}$ bajos, los cuales podrían ocasionar efectos nocivos contra los tejidos duros dentarios, 9,16 y sensibilidad postratamiento. 3

Los estudios se han centrado en evaluar el $\mathrm{pH}$ y las concentraciones de los agentes activos en relación a la eficacia blanqueadora y posibles efectos adversos de los productos.3,16 Sin embargo, a pesar de que se ha sugerido que el $\mathrm{pH}$ podría jugar un rol importante en la estabilidad química de la solución del peróxido de hidrógeno,12,13 a la fecha aún no hay estudios que intenten relacionar ambas variables y determinar de manera más precisa la influencia del $\mathrm{pH}$ en la concentración del peróxido de hidrógeno. Es por esto que realizar una evaluación de calidad de estos productos se vuelve un procedimiento necesario, ya que se espera mantengan las propiedades acordes a los estándares sugeridos por las normas desde el periodo de compra hasta el momento de su uso y más aún, hasta la fecha indicada de caducidad. Tal es así, que la misma norma ISO 28399 establece que la variación de concentración del agente activo no debe ser mayor a un $10 \%$ o menor a un $30 \%$ de lo especificado por el fabricante.

Por lo tanto, el objetivo del presente estudio in vitro, fue medir el pH y la concentración de peróxido de hidrogeno de geles blanqueadores de uso profesional disponibles en el mercado, y comparar dichos valores con los especificados por el fabricante.

\section{Materiales y Métodos}

Fueron seleccionados diferentes agentes blanqueadores de uso profesional cuyo agente activo es el Peróxido de Hidrógeno en altas concentraciones. Los productos seleccionados se detallan en la Tabla 1.

Una vez obtenidos los productos fueron almacenados siguiendo las indicaciones del fabricante. Las mediciones se realizaron siguiendo las normativas actuales para los productos de blanqueamiento.19,20 Todos los reactivos utilizados en el estudio fueron de calidad analítica y fueron preparados antes de iniciar cada experimento. Los productos se manipularon de acuerdo a las instrucciones de cada fabricante, con una temperatura del medio de 23 $\pm 2{ }^{\circ} \mathrm{C}$ según especificado en la normativa.

\section{Análisis de $\mathrm{pH}$}

La determinación del pH de los productos blanqueadores fue realizada conforme a las especificaciones que se encuentran establecidas en la norma ADA para la evaluación de agentes blanqueadores de consultorio.20 Previo a las mediciones, el medidor de $\mathrm{pH}$ (Beckman Zeromatic SS-3; Beckman Coulter Inc., Brea, CA, USA) fue calibrado utilizando soluciones tampón con niveles de $\mathrm{pH}$ de 4,7 y 10.

Para realizar las mediciones, se llenó un frasco pequeño de plástico con gel blanqueador previamente preparado conforma a las instrucciones del fabricante, lo suficiente como para cubrir la unión del electrodo. Se sumergió el electrodo dentro del gel blanqueador, asegurándose de que la punta y la unión del electrodo estuvieran completamente cubiertas, y sin burbujas de aire atrapadas contra el mismo. Se procedió a la lectura del pH luego de transcurrido 1 minuto. Para cada producto, se repitió la prueba cinco veces, usando para cada medición un kit de blanqueamiento diferente proveniente del mismo lote, totalizando cinco valores de $\mathrm{pH}$ para cada producto, registrándose los valores obtenidos. Para fines estadísticos, se registró el valor medio del pH para cada producto. 
Análisis de la concentración de peróxido de hidrógeno

La concentración de peróxido de hidrógeno en los productos blanqueadores fue realizada conforme a las especificaciones que se encuentran establecidas en la norma ADA para la evaluación de agentes blanqueadores de consultorio.20

En un matraz Erlenmeyer de $125 \mathrm{~mL}$ se pesaron $200 \pm 10$ $\mathrm{mg}$ de gel blanqueador y se agregaron $20 \mathrm{~mL}$ de agua desionizada hasta lograr la completa disolución. Luego, se añadió $20 \mathrm{~mL}$ de ácido sulfúrico $2,0 \mathrm{~N}$ y se mezcló con la ayuda de un agitador magnético. Posteriormente se llenó una bureta de $50 \mathrm{~mL}$ con $0.1 \mathrm{~N} \mathrm{KMnO}_{4}$ hasta la marca cero. Se agregó por goteo la solución con $\mathrm{KNnO}_{4} 0.1 \mathrm{~N}$ hasta que la misma cambió de clara a débil rosado. En ese momento se registró el volumen de $0.1 \mathrm{~N} \mathrm{KMnO}_{4}$ utilizado. Para realizar el cálculo de porcentaje $\mathrm{H}_{2} \mathrm{O}_{2}$ se utilizó la siguiente fórmula:

$$
\mathrm{H}_{2} \mathrm{O}_{2} \%=\frac{\text { Vol } \mathrm{KNnO}_{4} 0,1 \mathrm{~N}(\mathrm{ml}) \cdot 1 \cdot 701(\mathrm{mg}) \cdot 100}{\text { Cantidad del blanqueador }(\mathrm{mg})}
$$

Para cada producto, se repitió la prueba cinco veces, usando para cada medición un kit de blanqueamiento diferente proveniente del mismo lote, totalizando cinco valores de la concentración de $\mathrm{H}_{2} \mathrm{O}_{2}$ para cada producto. Para fines estadísticos, se registró el valor medio de la concentración de $\mathrm{H}_{2} \mathrm{O}_{2}$ para cada producto.

Tabla 1. Información comercial de los agentes blanqueadores utilizados

\begin{tabular}{|c|c|c|c|c|c|}
\hline Marca Comercial & $\begin{array}{c}\text { Componente } \\
\text { Activo* }\end{array}$ & Concentración* & $\mathrm{pH}^{*}$ & Almacenamiento* & Lote \\
\hline $\begin{array}{l}\text { Total Blanc H35 } \\
\text { (Nova DFL; Rio de } \\
\text { Janeiro, RJ, Brasil) }\end{array}$ & $\mathrm{H}_{2} \mathrm{O}_{2}$ & $35 \%$ & $\begin{array}{l}\mathrm{Si} \\
(2-4)\end{array}$ & 5 a $25^{\circ} \mathrm{C}$ & 16010110 \\
\hline $\begin{array}{l}\text { Whiteness HP Blue } \\
\text { (FGM Productos } \\
\text { Odontológicos; } \\
\text { Joinville, SC, Brasil) }\end{array}$ & $\mathrm{H}_{2} \mathrm{O}_{2}$ & $35 \%$ & $\begin{array}{c}\text { No } \\
\text { especificado }\end{array}$ & 5 a $25^{\circ} \mathrm{C}$ & 130515 \\
\hline $\begin{array}{c}\text { Pola Office } \\
\text { (SDI Limited; } \\
\text { Bayswater, VIC, } \\
\text { Australia) }\end{array}$ & $\mathrm{H}_{2} \mathrm{O}_{2}$ & $35 \%$ & $\begin{array}{c}\text { No } \\
\text { especificado }\end{array}$ & 2 a $25^{\circ} \mathrm{C}$ & $486 / 025141$ \\
\hline $\begin{array}{l}\text { Opalescence Boost } \\
\text { (Ultradent Products, } \\
\text { Inc; South Jordan, UT, } \\
\text { USA) }\end{array}$ & $\mathrm{H}_{2} \mathrm{O}_{2}$ & $40 \%$ & $\begin{array}{c}\mathrm{Si} \text { (indica pH } \\
\text { "neutro") }\end{array}$ & 2 a $8 \stackrel{\circ}{\circ}$ & BCD82 \\
\hline
\end{tabular}

$\mathrm{H}_{2} \mathrm{O}_{2}$ : Peróxido De Hidrógeno. pH: Potencial de Hidrogeniones. *Especificado por el fabricante.

\section{Análisis estadístico}

El estadístico $Z$ fue utilizado para comparar los valores promedio experimentales de $\mathrm{H}_{2} \mathrm{O}_{2}$ obtenidos de cada uno de los productos con el valor especificado por el fabricante. De igual forma, fueron comparados los valores experimentales de la concentración de $\mathrm{H}_{2} \mathrm{O}_{2}$ entre los diferentes productos blanqueadores a través de una prueba de Kruskal Wallis. El promedio de $\mathrm{pH}$ de los productos blanqueadores fue analizado a través de una prueba de Kruskal-Wallis.

\section{Resultados}

Los resultados de las mediciones de $\mathrm{pH}$ de los $A B$ se muestran en la Tabla 2. Se encontraron diferencias estadísticamente significativas entre los valores promedios de $\mathrm{pH}$ todos los productos analizados $(\mathrm{p}<0.001)$.

Tabla 2. $\mathrm{pH}$ de los geles blanqueadores

\begin{tabular}{cc}
\hline Producto & Promedio \\
\hline Pola Office & $2.78(0.41)^{\mathrm{c}}$ \\
Total Blanc H35 & $5.92(0.27)^{\mathrm{bc}}$ \\
Opalescence Boost & $7.08(0.19)^{\mathrm{ab}}$ \\
Whiteness HP Blue $^{\mathrm{ab}}$ & $8.26(0.15)^{\mathrm{a}}$ \\
\hline
\end{tabular}

Letras iguales en la columna del promedio indican que no existen diferencias significativas entre los grupos $(p<0.05$; prueba de Kruskal-Wallis)

Los resultados de las mediciones de $\mathrm{H}_{2} \mathrm{O}_{2}$ de los agentes blanqueadores se muestran en la Tabla 3 . Todos los 
grupos mostraron una disminución estadísticamente significativa en la concentración de $\mathrm{H}_{2} \mathrm{O}_{2}$ medida experimentalmente, con respecto a la especificada por su respectivo fabricante. Además, Opalescence Boost mostró una concentración de $\mathrm{H}_{2} \mathrm{O}_{2}$ experimental, significativamente mayor a los demás grupos.

\section{Discusión}

En el presente estudio in vitro, se evaluó y comparó el pH y la concentración de $\mathrm{H}_{2} \mathrm{O}_{2}$ de diferentes $\mathrm{AB}$ para uso profesional. Los resultados muestran productos con $\mathrm{pH}$ que oscilan desde una fuerte acidez, hasta una leve alcalinidad. Por otro lado, todos los productos evaluados presentaron concentraciones de $\mathrm{H}_{2} \mathrm{O}_{2}$ menores a las declaradas por el fabricante, aunque dentro de lo aceptable establecido en la norma ISO 28399.

Respecto al $\mathrm{pH}$ evaluado, en el presente estudio se observaron valores que variaron desde 2.5 hasta 8.5 (Tabla 2), siendo un intervalo mayor al reportado previamente en la literatura para productos de blanqueamiento de uso profesional, de 3.75 a 6.53.12 En este estudio, Pola Office (SDI Dental) presentó un pH promedio de 2.8, consecuentemente, este producto podría causar una mayor desmineralización del esmalte que los otros productos evaluados. Se ha demostrado que los dientes y tejidos orales sometidos a un $\mathrm{pH}$ bajo durante un período prolongado de tiempo pueden sufrir efectos secundarios adversos. Por ejemplo, la disminución del pH por debajo de 5.5 provoca una marcada desmineralización

Tabla 3. Concentración de $\mathrm{H}_{2} \mathrm{O}_{2}$ de los $\mathrm{AB}$ declarada por el fabricante y medida experimentalmente Información comercial de los agentes blanqueadores utilizados.

\begin{tabular}{|c|c|c|c|c|}
\hline Producto & Fabricante (\%) & Experimental (\%) & Test Z & $\begin{array}{l}\text { Porcentaje } \\
\text { variación* }\end{array}$ \\
\hline Pola Office & 35 & $30.1(0.35)^{a}$ & $\begin{array}{c}\text { Diferente al } \\
\text { estándar }\end{array}$ & $-11.54 \%$ \\
\hline Total Blanc H35 & 35 & $30.1(1.35)^{\mathrm{a}}$ & $\begin{array}{c}\text { Diferente al } \\
\text { estándar }\end{array}$ & $-11.6 \%$ \\
\hline $\begin{array}{c}\text { Whiteness HP } \\
\text { Blue }\end{array}$ & 35 & 27.2. $(2.44)^{\mathrm{a}}$ & $\begin{array}{c}\text { Diferente al } \\
\text { estándar }\end{array}$ & $-22.34 \%$ \\
\hline $\begin{array}{c}\text { Opalescence } \\
\text { Boost }\end{array}$ & 40 & $38.6(0.54)^{b}$ & $\begin{array}{c}\text { Diferente al } \\
\text { estándar }\end{array}$ & $-3.4 \%$ \\
\hline
\end{tabular}

del esmalte 21 y reabsorción radicular.22,23 En este sentido, las investigaciones sugieren que el $\mathrm{pH}$ bajo y las altas concentraciones de ácido causan la erosión del esmalte y que está erosión no es proporcional al tiempo de aplicación del ácido.24 Otros trabajos han evidenciado disminución de la microdureza del esmalte 25 y alteración en la composición mineral de $\mathrm{Ca}++$ y PO4-.26 Se ha sugerido un riesgo significativamente menor a la sensibilidad dental postratamiento producida por geles neutros, al compararlos con geles ácidos,3,17 sin embargo, no se han encontrado diferencias en el efecto blanqueante, demostrando ambos ser igual de eficaces. 17,27

Este último punto sugiere que la efectividad de los agentes blanqueadores está dada por los agentes químicos activos y no por la acidez del producto, 17,18 siendo los tiempos y las concentraciones aplicadas, responsables de los efectos blanqueadores.14,16,28 Se ha informado de que cuanto mayor es la concentración de peróxido, más ácido será el $\mathrm{pH}$ del producto blanqueador,23 sin embargo, esto no se condice con los resultados de este estudio, ya que productos con la misma concentración de $\mathrm{H}_{2} \mathrm{O}_{2}$ pueden presentar diferentes valores de $\mathrm{pH}$, inclusive $\mathrm{pH}$ neutro a una máxima concentración de $\mathrm{H}_{2} \mathrm{O}_{2}$ (Opalescense Boost, Ultradent). En este sentido, además del agente activo, el fabricante probablemente agregue agentes buffer no especificados ya que solamente se describen en su composición como neutralizantes.

Existen muy pocos estudios disponibles que determinen la concentración real de $\mathrm{H}_{2} \mathrm{O}_{2}$ en agentes de blanqueamiento dental para uso profesional.1,29 Este hecho dificulta la comparación de los resultados del 
presente estudio con datos de la literatura. Según nuestro conocimiento, ninguno de los agentes blanqueadores seleccionados en este estudio ha sido previamente evaluados. Los resultados muestran que todos presentaron una concentración de $\mathrm{H}_{2} \mathrm{O}_{2}$ menor a la declarada por el fabricante (Ver Tabla 3), con un porcentaje de 3.5 y hasta $22.3 \%$ menos de $\mathrm{H}_{2} \mathrm{O}_{2}$. Esto coincide con los estudios previamente mencionados, en donde se observó hasta un $27 \%$ menos de $\mathrm{H}_{2} \mathrm{O}_{2} .1$

Siendo el $\mathrm{H}_{2} \mathrm{O}_{2}$ una sustancia químicamente inestable, estas variaciones en la concentración podrían deberse a diversos factores, entre los cuales se pueden citar a la temperatura de almacenamiento y el pH del material. En relación a la temperatura de almacenamiento, se ha sugerido que un aumento de la misma fuera de los parámetros adecuados podría disminuir la eficacia blanqueadora 9 y el $\mathrm{pH}$ de los agentes blanqueadores. 13 Este hecho sugiere la importancia de almacenar los productos según lo indicado por el fabricante. En el presente estudio, 3 materiales fueron almacenados a temperatura ambiente y uno refrigerado, siguiendo las indicaciones de los fabricantes. Por otro lado, se pone en cuestionamiento el posible rol del $\mathrm{pH}$ en los geles, sugiriendo que la eficacia del $\mathrm{H}_{2} \mathrm{O}_{2}$ está directamente relacionado al mismo.10 Podría explicarse por el hecho de que la constante de disociación del $\mathrm{H}_{2} \mathrm{O}_{2}$ es alrededor de 11.5 , entonces, cuanto más básico sea el medio en el que se encuentra, más rápidamente se producirán las reacciones de óxido-reducción.30 Por lo tanto, productos con pH bajos podrían ser capaces de mantener por más tiempo la estabilidad del $\mathrm{H}_{2} \mathrm{O}_{2} .13$

Según los resultados de este estudio, el gel neutro $(\mathrm{pH}$ 7.08) fue el que presentó menor variación en la concentración de $\mathrm{H}_{2} \mathrm{O}_{2}$ respecto a la especificada por el fabricante $(-3.5 \%)$, seguido por los geles de $\mathrm{pH}$ levemente $(\mathrm{pH}$ 5.92) y fuertemente ácidos $(\mathrm{pH} 2.78)$ con una variación del $-11 \%$ con respecto a lo especificado por el fabricante, y finalmente el gel levemente básico $(\mathrm{pH}$ 8.26) que fue el que presentó una mayor variación de la concentración de $\mathrm{H}_{2} \mathrm{O}_{2}$, siendo de $-22.3 \%$ comparado con lo que especifica el fabricante. Estos resultados nos llevan a comprobar que un $\mathrm{pH}$ bajo es capaz de mantener por más tiempo la estabilidad del $\mathrm{H}_{2} \mathrm{O}_{2} .13 \mathrm{~A}$ pesar de las diferencias encontradas, es importante destacar que, aun así, todos los productos se encuentran dentro de los parámetros sugeridos por la normativa internacional, que especifica que los mismos pueden tener una disminución de hasta $30 \%$ de $\mathrm{H}_{2} \mathrm{O}_{2} .19$ Además, los $\mathrm{AB}$ con una concentración inicial o declarada de $35 \%$ de $\mathrm{H}_{2} \mathrm{O}_{2}$, a pesar de que presentaron valores de $\mathrm{pH}$ estadísticamente diferentes, no presentaron diferencias en cuanto a las concentraciones evaluadas.

En un estudio clínico previo, se comparó la eficacia blanqueadora entre el Whiteness HP Blue y el Pola Office, ambos con $35 \%$ de $\mathrm{H}_{2} \mathrm{O}_{2}$, mostrando tener una efectividad semejante,31 y sin embargo, según los datos del presente y de estudios previos, uno de ellos presenta $\mathrm{pH}$ levemente alcalino, y otro fuertemente ácido.32,33 Con esto, es posible hipotetizar que, el $\mathrm{pH}$ de un agente blanqueador, más que tener una influencia significativa en la capacidad blanqueadora, podría tener un efecto considerable en la estabilidad de las soluciones blanqueadoras. El tiempo de vida media o de anaquel de los productos podría verse influenciado además por la composición, por los métodos y condiciones de elaboración y por las condiciones de almacenamiento, esto último incluiría inclusive hasta el realizado por parte del odontólogo en su consultorio. Las pruebas aceleradas de vida en anaquel, las cuales rara vez se realizan para agentes blanqueadores, podrían ser objeto de futuras investigaciones para comprender los mecanismos de degradación de los componentes químicos presentes en agentes blanqueadores.

Los hallazgos del presente estudio sugieren que a pesar que los productos analizados presentaron diferentes valores de $\mathrm{pH}$, todos mostraron una reducción significativa en las concentraciones de $\mathrm{H}_{2} \mathrm{O}_{2}$ al compararlas con las declaradas por el fabricante. Estas podrían verse afectadas debido a un incorrecto transporte $y$ almacenamiento, lo que podría disminuir la calidad, eficacia y el tiempo de vida media de los productos.

Como futuros lineamientos, se propone una evaluación en la que se consideren diferentes momentos de las pruebas en relación a la fecha de vencimiento indicada por el fabricante. Además, dadas las particularidades de los $A B$ y la inestabilidad química de sus componentes activos, las pruebas de envejecimiento se vuelven necesarias a modo de intentar comprender mejor su calidad y vida media, por lo que más estudios en este campo deberían ser realizados.

\section{Conclusiones}

A pesar de las limitaciones, los resultados del presente estudio muestran que los Agentes Blanqueadores presentaron valores de $\mathrm{pH}$ que oscilaron desde una ligera alcalinidad hasta una fuerte acidez. En cuanto a las concentraciones de Peróxido de Hidrógeno, las mismas fueron inferiores a las especificadas por el fabricante. Las discrepancias observadas pueden afectar la eficacia de blanqueamiento esperada tanto por el paciente como por el dentista, y debe ser considerado a la hora de establecer un plan de tratamiento.

\section{Agradecimientos}

Los autores agradecen al ex Profesor de la Cátedra de Bioquímica de la Facultad de Odontología de la UdelaR, el Dr. Enrique Zinemanas, por su colaboración en el 
desarrollo de las metodologías realizadas en el presente trabajo.

\section{Referencias}

[1] Matis BA. Tray whitening: what the evidence shows. Compend Contin Educ Dent. 2003;24(4):354-62.

[2] Silva FB da, Chisini LA, Demarco FF, Horta BL, Correa MB Desire for tooth bleaching and treatment performed in Brazilian adults: findings from a birth cohort. Braz Oral Res. 2018;32:12.

[3] Loguercio AD, Servat F, Stanislawczuk R, Mena-Serrano A Rezende M, Prieto M V, et al. Effect of acidity of in-office bleaching gels on tooth sensitivity and whitening: a two-center double-blind randomized clinical trial. Clin Oral Investig. 2017 Dec;21(9):2811-8.

[4] Meireles SS, Goettems ML, Dantas RVF, Bona Á Della, Santos IS, Demarco FF. Changes in oral health related quality of life after dental bleaching in a double-blind randomized clinical trial. J Dent. 2014;42(2):114-21.

Demarco FF, Meireles SS, Masotti AS. Over-the-counter whitening agents: a concise review. Braz Oral Res. 2009 Jun;23(suppl 1):64-70.

Nie J, Tian FC, Wang ZH, Yap AU, Wang XY. Comparison of efficacy and outcome satisfaction between in-office and home teeth bleaching in Chinese patients. J Oral Sci. 2017;59(4):52732 .

[7] de Geus JL, Wambier LM, Kossatz S, Loguercio AD, Reis A. Athome vs In-office Bleaching: A Systematic Review and Metaanalysis. Oper Dent. 2016;41(4):341-56.

[8] Luque-Martinez I, Reis A, Schroeder M, Munoz MA, Loguercio $\mathrm{AD}$, Masterson $\mathrm{D}$, et al. Comparison of efficacy of tray-delivered carbamide and hydrogen peroxide for at-home bleaching: a systematic review and meta-analysis. Clin Oral Investig. 2016 Sep;20(7):1419-33.

[9] Chisini LA, Conde MCM, Meireles SS, Dantas RVF, Sarmento HR, Della Bona A, et al. Effect of temperature and storage time on dental bleaching effectiveness. J Esthet Restor Dent. 2019 Jan;31(1):93-7.

[10] Torres CRG, Crastechini E, Feitosa FA, Pucci CR, Borges AB Influence of $\mathrm{pH}$ on the effectiveness of hydrogen peroxide whitening. Oper Dent. 2014;39(6):E261-8.

[11] Al-Salehi SK, Hatton P V, McLeod CW, Cox AG. The effect of hydrogen peroxide concentration on metal ion release from dental amalgam. J Dent [Internet]. 2007;35(2):172-6. Available from: http://www.sciencedirect.com/science/article/pii/S03005712060 01333

[12] Price RB, Sedarous M, Hiltz GS. The $\mathrm{pH}$ of tooth-whitening products. J Can Dent Assoc. 2000 Sep;66(8):421-6.

[13] Freire A, Archegas LRP, de Souza EM, Vieira S. Effect of storage temperature on $\mathrm{pH}$ of in-office and at-home dental bleaching agents. Acta Odontol Latinoam. 2009;22(1):27-31.

[14] Kose C, Calixto AL, Bauer JRO, Reis A, Loguercio AD Comparison of the Effects of In-office Bleaching Times on Whitening and Tooth Sensitivity: A Single Blind, Randomized
Clinical Trial. Oper Dent. 2016;41(2):138-45.

[15] Sulieman M, MacDonald E, Rees JS, Newcombe RG, Addy M. Tooth bleaching by different concentrations of carbamide peroxide and hydrogen peroxide whitening strips: an in vitro study. J Esthet Restor Dent. 2006;18(2):93-100; discussion 101.

[16] Grazioli G, Valente LL, Isolan CP, Pinheiro HA, Duarte CG, Munchow EA. Bleaching and enamel surface interactions resulting from the use of highly-concentrated bleaching gels. Arch Oral Biol. 2018 Mar;87:157-62.

[17] Sa Y, Chen D, Liu Y, Wen W, Xu M, Jiang T, et al. Effects of two in-office bleaching agents with different $\mathrm{pH}$ values on enamel surface structure and color: an in situ vs. in vitro study. J Dent. 2012 Jul;40 Suppl 1:e26-34.

[18] Xu B, Li Q, Wang Y. Effects of $\mathrm{pH}$ values of hydrogen peroxide bleaching agents on enamel surface properties. Oper Dent. 2011;36(5):554-62.

[19] International Organization for Standardization. ISO 28399: Dentistry — Products for external tooth bleaching. 2011.

[20] American Dental Association. In-Office Whitening Agents: Laboratory Testing Methods. 2008.

[21] Meurman JH, ten Cate JM. Pathogenesis and modifying factors of dental erosion. Eur J Oral Sci. 1996 Apr;104(2 ( Pt 2)):199206

[22] Rotstein I, Friedman S, Mor C, Katznelson J, Sommer M, Bab I. Histological characterization of bleaching-induced external root resorption in dogs. J Endod. 1991 Sep;17(9):436-41.

[23] Weiger R, Kuhn A, Löst C. Effect of various types of sodium perborate on the $\mathrm{pH}$ of bleaching agents. J Endod [Internet]. 1993;19(5):239-41. Available from: http://www.sciencedirect.com/science/article/pii/S00992399068 12999

[24] Hunter ML, West NX, Hughes JA, Newcombe RG, Addy M. Relative susceptibility of deciduous and permanent dental hard tissues to erosion by a low $\mathrm{pH}$ fruit drink in vitro. J Dent. 2000 May;28(4):265-70.

[25] Abouassi T, Wolkewitz M, Hahn P. Effect of carbamide peroxide and hydrogen peroxide on enamel surface: an in vitro study. Clin Oral Investig. 2011;15(5):673-80.

[26] Roselino L de MR, Chinelatti MA, Alandia-Roman CC, Pires-deSouza F de CP. Effect of Brushing Time and Dentifrice Abrasiveness on Color Change and Surface Roughness of Resin Composites. Braz Dent J. 2015 Oct;26(5):507-13.

[27] Sun L, Liang S, Sa Y, Wang Z, Ma X, Jiang T, et al. Surface alteration of human tooth enamel subjected to acidic and neutral 30\% hydrogen peroxide. J Dent. 2011 Oct;39(10):686-92.

[28] Bersezio C, Estay J, Jorquera G, Pena M, Araya C, Angel P, et al. Effectiveness of Dental Bleaching With $37.5 \%$ and $6 \%$ Hydrogen Peroxide and Its Effect on Quality of Life. Oper Dent. 2019;44(2):146-55.

[29] Majeed A, Farooq I, Grobler SR, Moola MH. In vitro evaluation of variances between real and declared concentration of hydrogen peroxide in various tooth-whitening products. Acta Odontol Scand. 2015;73(5):387-90. 
Publicación semestral, Educación y Salud Boletín Científico Instituto de Ciencias de la Salud Universidad Autónoma del Estado de

Hidalgo, Vol. 8, No. 16 (2020) 1-7

[30] Frysh H, Bowles WH, Baker F, Rivera-Hidalgo F, Guillen G. Effect of $\mathrm{pH}$ on hydrogen peroxide bleaching agents. J Esthet Dent. 1995;7(3):130-3

[31] Matis BA, Cochran MA, Franco M, Al-Ammar W, Eckert GJ, Stropes M. Eight in-office tooth whitening systems evaluated in vivo: A pilot study. Oper Dent. 2007;32(4):322-7.

[32] Trentino AC, Soares AF, Duarte MAH, Ishikiriama SK, Mondelli RFL. Evaluation of $\mathrm{pH}$ Levels and Surface Roughness After Bleaching and Abrasion Tests of Eight Commercial Products. Photomed Laser Surg. 2015 Jul;33(7):372-7.

[33] Lilaj B, Dauti R, Agis H, Schmid-Schwap M, Franz A, Kanz F, et al. Comparison of Bleaching Products With Up to $6 \%$ and With More Than 6\% Hydrogen Peroxide: Whitening Efficacy Using B and WI (D) and Side Effects - An in vitro Study. Front Physiol [Internet]. 2019 Aug 21;10:919. Available from: https://pubmed.ncbi.nlm.nih.gov/31543822 